\title{
ON BEHAVIOR OF SOLUTIONS OF NON-LINEAR DIFFERENTIAL EQUATIONS IN HILBERT SPACE II
}

\author{
VLADIMIR SCHUCHMAN \\ Lefariment of Mathematics \\ North Texas State li:iversity \\ Denton, iexas 76203 USA \\ (Received December 30, 1981 and in revised form April 12, 1984)
}

\begin{abstract}
This paper deals with the behavior of solutions of non-linear ordinary differntial. equations in a Hilbert space with applications to non-linear partial differential equations.
\end{abstract}

KEY WORDS 3 HJD PHRASES. Nonlinear differential equations, self-adjoint equations, non-degenerate equations and solution.

1980 AMS SUBJECT CLASSIFICATION CODE. 16A70

I. INTRODUCTION.

ill this paper, we contirue nur study of behavior of sclutions of non-lineior ordinary differential equations ir. a Hilbert space $H$ with applications to nonlinear partial differeritial equations.

We introduce here ncn-linear cperator of self-adjoint type and we study the cuasiuniqueress of Calichy problen and the classical uniqueness of Cauchy problem for this equation.

In the special case unaer condition A we obtain complete results abcut quasiuniqueness. Recall that we do rot have this situation in a linear case because this part of our thecrems have nu arialog in the linear case.

As usual, we study twc cases of non-linear differential equations: the case of decenerate equation in bounded interval of time and the case cf non-degenerate equation ir. unbounded interval of time.

In the firit part of this faper, we study the following non-linear degenerate equation:

$$
t \frac{d u}{d t}=B(t, u(t))
$$

where $t \in I=(0,1]$. $u(t)$ for each $t \varepsilon I$ is an element of $H$ and has derivative with respect to $t$, if $t>0$. $B(t, u(t))$ is a non-linear map from $H$ to $H$ with domain $D_{B}, D_{B}$ is the dense subset of $H$ and for each $t \varepsilon I$ anc $u(t) \varepsilon$ $D_{E}, B(t, u(t))$ is an elenent of $H$ also. $B(t, u(t))$ is not necessarily bounded. Special cast of equation $(*)$ is the case $(1.20)$, that $B$ is a frocuct of the form

$$
B(t, u(t))=A(t, u(t)) \cdot u(t)
$$

where $A(t, u(t))$ is a non-linear map from $H$ to $H$. In this situation, we obtain complete resuits about the quasiuniqueness at the foint $t=0$. Our Theoreri? $? .3$ is the nia in theorer of the first part. 
The: anr-degenerate equation which we alse : lway is thic couation of the torm

$$
\frac{d u}{d t}=P(t, u l: i
$$

ihere $t=\bar{I}=[1,+\infty)$. $a(t)$ fur edch $t \in \bar{I}$ is ar. Eiement of $H$ and has derivative wit! respect to $t .[(t, u(t))$ is a ron-linear map from $H$ to $H$ with dumain $D_{E}, D_{B}$ is the dense subcet of $H$ and for fach $t \in \bar{I}$ and $u(t) \varepsilon D_{B}, B(t, u(t))$ is dil eleneil uf $t_{i}$ also. $B(r, u(t))$ is not recessarity bounded.

We obtain complete results for the salie sfecial case as above. The rila in theorem of this part of uur study is Theorem 4.7 abclit the nuasiuniqueness of equation (**) in a special cast at the point $t=+a$.

In $\S 1$ we study the quis iuniquenes for equation $\left(^{*}\right)$. In $\$ \hat{2}$ we study the quasiuniqueress in two special cases and we obtain complete Theorem 2.3 in the special case (1.20) of equation (*). in $\$ 3$ we study the uniqueness of Cauchy froblem for special cast of equation $(*)$. We chtain partid? results only for this problem. In. 84 we study ncr-degenerate equation (**) and we olitain for ihis equation the same results as for ectuation (*). The Theoren 4.7 is a parallel to Theorem 2.3 . In $\notin 5$ we study several examples of non-innear partial differential equations with cordition (1.2n) and we obtain for there equations, the quasiuriqueness at the point $t=0$ in the desenerate cuse and at the pcint $t=+\infty$ in the non-degentrate case. Recall that we have no aralog of these theorems ir, a linear case.

The metricd of this study was used first by Agnion-Nirenberg $[1,2]$ for studying the classical uniqueness of Cauchy problem in the non-degenerate linear case. This nethod in the degenerate case was used by the author in [3].

This methoc was used by the author fur study of the quasiuniqueness in the ron-linear case for the following special equation:

$$
t \frac{\partial u}{\partial t}=\frac{\partial}{\partial x} K(u) \frac{\partial u}{\partial x}
$$

and for the rion-degenerate equation of the similar type [4]. Several theorems of this paper are like thecrems of paper [4], but here we have the case of non-linear equation, and in [4] we studied special case of quasilinear equations. Several theorems for example, Theorem 2.1 or Theorem 4.5, were obtaineo in paper [5] also. $\S 1$. On the quasiuniqueness in degenerate case, let us consider the following nonlinear equation in the Hilbert space $H$.

$$
t \frac{\partial u}{\partial t}=B(t, u(t))
$$

where $t \varepsilon I=(0,1]$. $u(t)$ for each $t \varepsilon I$ is an element of $H$ and has derivative with respect to $t$, if $t>0$.

$B(t, u(t))$ is a non-linear map from $H$ to $H$ with domain $D_{B}$, $D_{B}$ is the dense subset of $H$, and for each $t \in I$ and for each $u(t) \varepsilon D_{B}, B(t, u(t))$ is an element of $H$ also. $B(t, u(t))$ is not necessarily bounded. $H$ is a Hilbert space with scalar product $(.,$.$) and with norm \|$.$\| correspondingly.$

Definition 1.1. The non-linear operator $B(t, u(t))$ is called snooth operator of self-adjoint type if the foliowing condition is satisfied:

Condition $S$. For each $t \in I$ and for each $u(t) \in D_{B}$ the following scalar product

$$
(B(t, u(t)), u(t))
$$

is real and differentiable with respect to $t$ if $t>0$. We study in this paper the behavior of the norm of the solutions of equation (1.1) under Condition $S$ only. 
Definition 1.2. Let $f(t)$ be a scalar function in interval $1 . f(t)$ is called a flat at the point $\tau=0$, if for each $k>0$

$$
t^{-K_{f}} f(t)+0 \text { as } t \rightarrow 0 \text {. }
$$

[efinition 1.3. The solution $u(t)$ of equation (1.1) is called a flat solution, if $\|u(t)\|$ is a flat function at the point $t=0$. The question which we have now is when equation (1.1) has no flat solutions. In the linear case with self-adjoint operator $B(t)$, the followirig statement is true.

Theorem 1.1. Let $B(t)$ be a linear symmetric operator with domain $D_{B}(t)$ and

$$
\frac{d}{d t} B(t) x=B(t) x \text { for each } x \in D_{B}(t) \cdot
$$

Let $u(t)$ be a solution of the following equation

$$
t \frac{d u}{d t}=B(t) u
$$

such that one of the following conditions is satisfied:

or

$$
\|\dot{B}(t) u(t)\| \leq \gamma(t)|| B(t) u(t)\|+B(t)|| u(t)\|
$$

$$
(\dot{B}), u(t)) \geq-\gamma(t)|(B(t) u(t), u(t))|-B(t)|| u(t) \|^{2}
$$

where $\gamma(t), B(t)$ are non-negative continuous functions in the interval $I^{\prime}=[0,1]$. Then for this solution $u(t)$, the following is true:

i) $\|u(t)\| \geq M\|u(t)\| t^{\nu+\mu}$

where constant $\nu \geq 0$ depending on $v(t), \nu(t)$ and $u(t)$ itself and constant $\mu \geq 0$ depending on $\nu(t), \beta(t)$ only.

ii) if $u(t)$ is a flat solution of equation $(1.4)$, then $u(t) \equiv 0$ in the interval $\mathrm{I}$.

Proof. (See [3]).

In the linear case, we do not have classical uniqueness at the point $t=0$, only the type of uniqueness as in $i i$ ) above--the quasiuniqueness (see Definition 1.4 below).

Definition 1.4. We say that the quasiuniqueness takes place for equation (1.1) or (1.4) at the point $t=0$ if conclusion $i i)$ of Theorem 1.1 is true for neighborhood of the point $t=0$ or, in other words, if we have uniqueness in the class of flat-functions at the point $t=0$. Let $u(t)$ be a solution of (1.1). Let

$$
q(t)=(u(t), u(t)) \text {. }
$$

If $t \frac{d}{d t}=D$, we have from (1.1) after scalar product with $u(t)$

$$
D q(t)=2 \operatorname{Re}(b(t, u(t)), u(t))=2(B(t, u(t)), u(t))
$$

and if we assume that $u(t) \varepsilon C^{1}(I, H)$, and $B(t, u)$ has first derivative with respect to all variables (see Condition $S$ ), then

$$
\begin{aligned}
D^{2} q & =2 D(B(t, u(t)), u(t))=2[([D B(t, u(t))], u(t))+(B(t, u(t)), D u(t))] \\
& =2[([D B(t, u(t))], u(t))+(B(t, u(t)), B(t, u(t)))] \\
& =2\left(\left[D_{B}(t, u(t))\right], u(t)\right)+2|| B(t, u(t)) \|^{2}
\end{aligned}
$$

Let now $\left(t_{1}, t_{0}\right)$ be the interval with $q(t)>0$ for $t \varepsilon\left(t_{1}, t_{0}\right)$, then if

$$
\ell(t)=\ln q(t) \text {, }
$$

then from (1.9) we have

$$
D \ell(t)=\frac{D q}{q}=\frac{2(B(t, u(t)), u(t))}{q(t)}
$$


and

or from (1.9) and (1.10) we obtain

$$
D^{2} s(t)=\frac{r^{i} G}{a}-\left(\frac{D q}{q}\right)^{2}
$$

$$
D^{2} \varepsilon(t)=\frac{2([U B(t, u(t))], u(t))}{q(t)}+\frac{2\left\lfloor B(t, u(t)) \|^{2}\right.}{q(t)}-\frac{4(B t, u(t)), u(t))^{2}}{q^{2}(t)}
$$

The followirig statement is true:

Lemma 1.1. Let $s(t)$ be a twice differentiable function in the interval $i$ satisfying the following second-order differential inequality

$$
D^{2} \ell(t)+t a(t)|D C(t)|+t b(t) \geq 0 . \quad t \in 1
$$

where $a(t), e(t)$ are non-negative bounded functions in $I$.

Then

$$
\ell(t) \ell \geq\left(t_{0}\right)+2 v \ln \frac{t}{t_{0}}+2 u \ln n_{\frac{t}{t}} \frac{t}{0}
$$

where constant $v>0$ depending on $a(t), b(t)$ and $1(t)$ itself, and constant $\mu>0$ depending on $a(t), b(t)$ only.

Prouf. (see $[3,4]$ ).

Fron (1.16) we obtain

$$
\exp \ell(t) \geq\left[\operatorname{expl}\left(t_{0}\right)\right] \cdot t^{2 v+2 \mu}
$$

and from definition $\ell(t)$ we have

$$
q(t)=\operatorname{expl}(t), q\left(t_{0}\right)=\operatorname{expl}\left(t_{0}\right)
$$

and from this and from (1.17) we have the following estimate for $q(t)$

$$
q(t) \geq q\left(t_{0}\right) t^{2 v+2 \mu}
$$

where corrstant $\nu>0$ depending on $a(t), b(t)$, and $q(t)$ itself, and constant $\mu>0$ deperiding on $a(t), b(t)$ only.

From (1.18) we obtain estimate (1.7). Our problem now is to obtain the inequality of type (1.15) for non-linear equation (1.1). From this discussion we obtain that the following statement. is true.

Theorem 1.2. Let $u(t)$ be a solution of equation (1.1) such that

$$
\begin{gathered}
\left([C B(t, u(t)], u(t))+\|B(t, u(t))\|^{2}-\frac{2(b(t, u(t)), u(t)))^{2}}{(u(t), u(t)))} \geq\right. \\
-t a(t)\|(B(t, u(t)), u(t)) \mid-t b(t)\| u(t) \|^{2}
\end{gathered}
$$

for some non-negative bounded functions $a(t), b(t)$ in the interval $I$. Then

i) $\|u(t)\| \geq M\left\|u\left(t_{0}\right)\right\| t^{v+\mu}$ where constant $\nu \geq 0$ depending on $a(t), b(t)$ and $u(t)$ itself, and constant $\mu \geq 0$ depending on $a(t), b(t)$ only.

ii) if $u(t)$ is a flat solution, then $u(t) \equiv 0$ in the interval I.

Proof. i) follows from Lemma 1.1 and $i i)$ follows immediately from $i$ ). Let us consider the special case of operator $B(t, u)$. Let Hilbert space $H$ satisfy the following condition: for each $u, v \in H, u \cdot v \in H$ also. Let us now consider that $B(t, u(t ;)$ is the product of the following form:

$$
B(t, u(t))=A(t, u(t)) \cdot u(t)
$$

where $A(t, u(t))$ is a non-linear map from Hilbert space $H$ to $H$ with doma in $D_{A}$, $D_{A}$ being the dense subset of $H$, and $A(t, u(t))$ satisfies the following condition: Condition $A$. For each $u(i) \in D_{A}$ and for each $v \in H$ the following function 
$(A(\tau, u(t)) v, v)$ is difterentiable with respect to $t$ and for each $w \varepsilon H$, $\left(A(t, u(t), v, w)=(v, A(t, u(t)) w)\right.$. In a standaru case $H$ is $L_{2}$ on compact set of $R^{n}$ or compact smicoth $n$-manifcld. In applications we rave $A(t, u)$ in the following form:

$$
A(t, u)=F\left(t, x, \frac{\partial u}{\partial x_{1}}, \ldots, \frac{\partial^{m} u}{\partial x^{m}}\right)
$$

where $x \in s 2, s 2$ is compact set of $R^{n}$ or smooth compact $n$-manifold (for example, sphere $s_{r}$.

Ir this special case, it is possible to obtain more complete results and more simple form of condition (1.19). Namely, it is possible to rewrite the first term of (1.19) in the following form:

$$
\begin{gathered}
([D B(t, u(t)], u(t))=([D A(t, u) u], u)=([D A(t, u)] u, u)+(A(t, u) D u, u)= \\
([D A(t, u)] u, u)+(D u, A(t, u) u)
\end{gathered}
$$

And from (1.1) aric $(1.20)$ we have that the lust term is

$$
\|A(t, u(t)) u(t)\|^{2}
$$

or

From this and (1.19) we obtain

$$
\|B(t, u(t))\|^{2}
$$

$$
\begin{gathered}
([D A(t, u)] u, u)+2\|A(t, u(t)) u\|^{2}-\frac{2\left(A(t, u(t) u, u)^{2}\right.}{(u(t), u(t))^{2}} \\
\geq-t a(t) \mid\left(A \left(t, u(t) u(t), u(t) \mid-t b(t)\|u(t)\|^{2}\right.\right.
\end{gathered}
$$

and since

$$
|(A(t, u(t)) u(t), u(t))| \leq\|A(t, u(t)) u(t)\| \cdot\|u(t)\|,
$$

we obtain that (1.22) is satisfied, for example, if the following condition is satisfied:

$$
([D A(t, u(t))] u(t), u(t)) \geq-t a(t)|(A(t, u(t)) u(t), u(t))|-t b(t)\|u(t)\|^{2}
$$

or, since $D=t \frac{d}{d t}$, after dividing on $t$ :

$$
\left(\left[\frac{d}{d t} A(t, u(t))\right] u(t), u(t)\right) \geq-a(t)|(A(t, u(t)) u(t), u(t))|-b(t)\|u(t)\|^{2} \text {. }
$$

This condition is very similar to condition (1.6) in the linear case. From the previous discussion we obtain that the following statement is true.

Theorem 1.3. Let $u(t)$ be a solution of equation (1.1) under Condition A such that condition (1.24) is satisfied for some non-negative bounded functions $a(t)$, $b(t)$ in the intervar $I$.

Then:

$$
\text { i) }|| u(t)\|\geq M\| u\left(t_{0}\right) \|^{v+\mu}
$$

where constant $\nu \geq 0$ depending on $a(t), b(t)$ and $u(t)$ itself, and constant $\mu \geq 0$ depending on $a(t), b(t)$ only.

ii) if $u(t)$ is a flat solution, then $u(t) \equiv 0$ in the interval I. Proof. i) follows from previous discussion and Lenma 1.1 and $i i)$ follows immediately from estimate (1.25).

Remark 1.1. Our condition (1.24) is not simple enough, and in concrete situations, it is difficult to check it. But in the following special case, when 


$$
(A(t, u(t)) v, v) \geq C \text { fer each } \vee \varepsilon H,
$$

it is possible to write other ccricitions in the followirg form.

If $(A(t, u) v, v)$ as a function of $t$ satisfifs the following condition for soluition $u(t)$ of ecuation (1.i):

$$
\left.\frac{d}{d t}\left[\left(A^{\prime}, t, u\right) v, v\right)\right] \geq-C(A(t, u(t)) v, v)
$$

for some constant $C>0$, and for each $v,\|v\|=1$, then for this solution $u(t)$, the conclusion of Theorem 1.3 is irue.

It is pcssible to obtain the following siatement abuut the quasiuniqueness.

Theorem 1.4. Let conditions $A$ and $(1.26)$ he satisfied. Let condition (1.27) be satisfied for each function $u(t) \in D_{A}$ with flat norm $\|u(t)\|$. Let $u(t)$ te a flat sciution of equation (1.1). Thell $u(i) \equiv 0$ in the interval $I$. Or, in other worc's, under these conditions, the atisiuniqueriess takes place at the pornt $t=0$ for sulutions of equation (1.1).

Proof. Let $u(t)$ be a flat solution. Then for $u(t)$, condition (1.27) is satisfied, and therefore for $\ell(t)=\ln \|u(t)\|^{2}$ we have ineqlality (1.15). From Lemria 1.1 , we have for $\|u(t)\|^{2}$ an estimate of the type (1.18). This is a contraciiction with flatness $u(t)$. Therefore, $u(t)$ is a trivial solution in the interval $I$, or the quasiuniqueness takes place at the point $t=0$ for solutions of equation (1.1) under conditions $A,(1.26),(1.27)$.

Remark 1.i. Let $f(t)$ be a scalar function in the interval I. Let $f(t)$ satisfy the following condition

$$
f^{\prime}(t) \geq-c f(t)
$$

or

$$
f^{\prime}(t)+c f(t) \geq 0 \text {. }
$$

It is possible to rewrite this condition in the following form

$$
\left[e^{c t} f(t)\right]^{\prime} \geq 0 \text {. }
$$

In other words, the following function

$$
e^{c t} f(t)
$$

is monotonic and not decreasing in the interval $I$. It is easy to see that each furction $f(t)$ will satisfy this condition in the interval $(0, \varepsilon)$, if

i) $f(0)>0$

ii) $f^{\prime}(0)$ is bounded.

Interval $(0, \varepsilon)$ depends on function $f(t)$.

From this discussion, we obtain that the following statement is true.

Iheorem 1.5. Let conditions $A$ and $(1.26)$ be satisfied. Let $A(t, u)$ for each $u(t) \varepsilon C_{A}$ with flat norm $\|u(t)\|$ satisfy the following conditions:

i) $(A(t, u(t)) v, v)>0$ in some interval $(0, \varepsilon)$ with $\varepsilon$ depending on $u(t)$ for each $\|v \varepsilon H\|, v \|=1$.

ii) $\frac{d}{d t}[(A(t, u(t)) v, v)]$ is bounded in interval $(0, \varepsilon)$ for each $v \varepsilon H,\|v\|=1$ (this condition follows from Conaition $A$, ).

If $u(t)$ is a flat solution of equation (1.1), then $u(t) \equiv 0$ in the interval $(0, \varepsilon)$ with $\varepsilon$ depending on $u(t)$, or the quasiuniqueness takes place at the point $t$ $=0$ for solutions of equation (1.1) under those conditions.

$\S 2$. The special cases

In this § we study two special cases for equation of type (1.1) for completing

Theorem 1.5. The first special case is the following: 


$$
\begin{array}{r}
(B(t, u), u(t))=0 \\
\text { fri each } u(t) \text { from the domain } D_{B} \text { In this case, if } \\
q(t)=\| u(t) i_{1}^{2}
\end{array}
$$

then

$$
\dot{t} \dot{q}(t)=2(B(t, u), u) \leq 0
$$

or

$$
q(t) \geq q\left(t_{0}\right) \text { for } t \leq t_{0}
$$

and we obtain that the following statement is true. Theorem 2.1. Let $u(t)$ be a solution of equation (1.1) under condition (2.1). If $u(0)=0$, then $u(t)=0$ in the interval $I$. In this situation, we have classical uniqueriess at the point $t=0$. kemark 2.1. Let operator $B(t, u)$ satisfy condition $A$ and let $A(t, u)$ satisfy the following condition:

$$
[(A(t, u(t)) v, v)] \leq 0
$$

ior each $u(t) \varepsilon \square_{A}$ with the flat $\|u(t)\|$ in the interval $[C, \varepsilon]$ with $\varepsilon>0$ depending on $u(t)$ may be and for each $v \varepsilon H,\|v\|=1$. Let $u(t)$ be a flat solution of equation (1.1) under those conditions. Then $u(t) \equiv 0$ in the interval $[0, \varepsilon]$ or the quasiuniqueress take place at the point $t=0$ for solutions of equation (1.1) under those conditions.

The second special case is the following:

Let $B(t, u)$ satisfy condition $A$ and let $A(t, u)$ as a function of $t$ satisfy the following condition:

$$
(A(t, u) v, v) \rightarrow C \text { as } t \rightarrow 0
$$

for each $u(t)$ with flat norm and for each $v \varepsilon H$ with $\|v\|=1$.

Let $q(t)=(u(t), u(t))$. If $q(t)>0$ in the interval $\left(t_{1}, t_{0}\right)$ we introduce a new function by formula

$$
v(t)=\frac{u(t)}{q \frac{1}{2}(t)}
$$

or

$$
u(t)=q \frac{1}{2}(t) v(t)
$$

After scalar produce (1.1) with, $u(t)$ we obtain

$$
t \dot{q}=\hat{c}\left(\left(A\left(t, q \frac{1}{2} v\right) u, u\right)=2 q\left(A\left(t, q \frac{1}{2} v\right) v(t), v(t)\right)\right. \text {. }
$$

Let now $u(t)$ be a solution of (1.1) under condition $A$ and $A(t, u)$ satisfies condition (2.3). The following scalar product

$$
\left|\left(A\left(t, q \frac{1}{2} v\right) v(t), v(t)\right)\right|<\delta
$$

in some neighborhood of the point $t=0$. This neighborhood depends on a solution $u(t)$ itself. From this we have that for each $u(t) \varepsilon D_{A}$ with flat norm $q(t)$ there exist numbers $\varepsilon, \delta>0$, such that

$$
\left|\left(A\left(t, q \frac{1}{2} v\right) v(t), v(t)\right)\right|<\delta \text { for } 0<t<\varepsilon
$$

where $\delta$ depends on $u(t)$.

From this we have for $q(t)$ the following inequality:

$$
\frac{t \dot{q}}{q}<2 \delta \text { for } 0<t<\varepsilon
$$

where $\delta$ depends on $u(t)$. From (2.5) we have the following estimate for $q(t)$

$$
q(t)>\left(\frac{t}{t_{0}}\right)^{2 \delta} q\left(t_{0}\right) \text { for } t<t_{0}<\varepsilon
$$


and trom this estimate, we have the quasiuniqueness. From the previous discussion we obtain that the following statement is true.

Theorem 2.2. Let $A(t, u)$ satisfy the following condition:

$$
(A(t, u) v, v) \rightarrow 0 \text { as } t \rightarrow 0
$$

for each $u(t) \in D_{A}(t)$ with flat rorm and for each $v \varepsilon H$ with $\|v\|=1$. Then if $u(t)$ is a flat solution of equation (1.1) under conditions $A$ and (2.6), then $u(t)$ is a trivial in the interval $(G, \varepsilon)$ with $\varepsilon>0$ depending on $u(t)$ itself.

From Theorems 1.5, 2.1, and 2.2, we have that the following statement is true.

Theorem 2.3. Let operator $B(t, u(t))$ satisfy coridition $A$. Then the quasiuniqueness takes place at the point $t=0$ for suiutions of equation (1.1) under those conditions.

Proof. Let $u(t)$ be a flat sclution of (1.1). Let us consider the following function:

$$
(A(t, u(t)) u(t), u(t))=q(t)(A(t, u(t)) v(t), v(t))
$$

where

and

$$
q(t)=\|u(t)\|^{2}
$$

$$
u(t)=v(t) q \frac{1}{2}(t) \quad\|u(t)\|=1 \text {. }
$$

It follows from condition $A$ that the following function

$$
f(t)=(A(t, u(t)) v(t), v(t))
$$

is continuous and smocth in the same interval $(0, \varepsilon)$ with $\varepsilon$ depending on $u(t)$. Then we have three cases:

$$
\begin{aligned}
\text { i) } f(t) & \leq 0 \text { in some interval }\left[0, \varepsilon^{\prime}\right] \text {. } \\
\text { ii) } f(t) & >0 \text { in some interval }\left[0, \varepsilon^{\prime}\right] \text {, } \\
\text { iii) } f(t) & >0 \text { as } t+0 .
\end{aligned}
$$

The quasiuniqueness follows for the case $i)$ from Remark 2.1, for the case $i i$ ) from Theorem 1.5, for the case $i i i)$ from Theorem 2.2 .

Remark 2.2. Smoothness in the Theorem 2.3 is necessary for the quasiuniqueness. In the finite-dimension Hilbert space, the smoothness also is necessary. Let us consider the following equation:

$$
t \frac{d x}{d t}=-x \ln x
$$

in the interval $I=(0,1]$. It is easy to see that the following function

$$
x(t)=\left\{\begin{array}{l}
\exp \left(-\frac{1}{t}\right) t>0 \\
0 \\
t \leq 0
\end{array}\right.
$$

is a solution of equation (2.7) for all $t \in R^{1}$. This function is flat and $x(t) \varepsilon$ $C^{\infty}\left(R^{1}\right)$. More than this, let us consider the following first order non-linear inequality

$$
\operatorname{tq}(t) \geq-C q(t) \ln q(t) \quad q(t) \geq 0
$$

and we will look for the solutions with $q(t)<1$ for $0<t<\delta$ only. It is possible to rewrite inequality (2.9) in the following form

$$
t \dot{q}(t)+C q(t) \ln q(t)=\phi(t) \geq 0 \text {. }
$$

Let

$$
1(t)=-\ln q(t)
$$

and 
$l(t) \geq 0$.

After dividing ( 2.10$)$ over $-q$ we obtain

$$
-\frac{t \dot{q}}{q}-\operatorname{cin} q(t)=-\frac{\phi(t)}{q(t)} \leq 0
$$

or after substitution (2.11) we obtain from (2.12) the following inequality for $1(t)$

$$
t 1(t)+C 1(t)=-\frac{\phi(t)}{q(t)} \leq 0
$$

Now we introduce a new function $m(t)$ by formula

$$
m(t)=\ln 1(t) \text {. }
$$

This function is defined, since $1(t) \geq 0$. From (2.13) we oblain for $m(t)$

$$
\dot{t} \dot{1}(t)+C=\phi_{1}(t) \leq 0
$$

aria

After integrating (2.15) we get

$$
\phi_{1}(t)=\frac{-\phi(t)}{C(t) q(t)}
$$

$$
m(t)-m\left(t_{0}\right)=-\operatorname{cln} \frac{t}{t_{0}}+t_{0}^{t} \phi_{1}(\tau) d \tau
$$

or

$$
\frac{1(t)}{1\left(t_{0}\right)}=-\operatorname{cln} \frac{t}{t_{C}}+t_{0}^{t} \phi_{1}(\tau) d \tau
$$

From (2.18) we obtain for $1(t)$

$$
r(t)=1\left(t_{0}\right)\left(\frac{t}{t_{0}}\right)^{-C} \exp t_{0}^{s} \phi_{1}(\tau) d \tau
$$

and from (2.11) and (2.19) we obtain for $q(t)$

$$
\ln q(t)=-1\left(t_{0}\right)\left(\frac{t}{t_{0}}\right)-C^{t} t_{0} t_{0}^{\delta \phi_{1}}(\tau) d \tau
$$

or for $q(t)$ we have

$$
q(t)=\exp \left[-1\left(t_{0}\right)\left(\frac{t}{t_{0}}\right)^{-C} \exp t_{0}^{t} t_{0}^{\left.\delta \phi_{1}(\tau) d \tau\right]}\right.
$$

In (2.20) $1\left(t_{0}\right)>0$ and $-1\left(t_{0}\right)<0$. The following integral

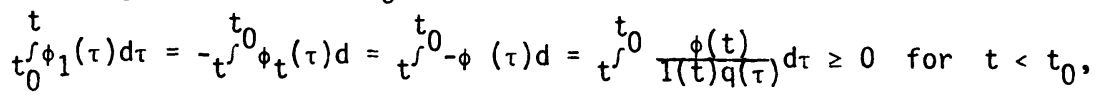

since $\phi(t) \geq 0,1(t) \geq 0, q(t) \geq 0$. From this we have that

$$
\exp t_{0}^{t} \phi_{1}(\tau) d \tau \geq 1
$$

and since $-1(t)<0$, then we have from (2.21) and (2.22) that the following estimate holds

$$
q(t) \leq \exp \left(-1\left(t_{0}\right)\left(\frac{t}{t_{0}}\right)^{-C}\right)=x(t)
$$

Function $x(t)$ in (2.23) is a flat and from this discussion we obtain that the following statement is true.

Theorem 2.4. Let $q(t)$ be a solution of inequality (2.9) with the condition

$$
q(t) \leq 1 \text { for } 0<t<\delta .
$$

Then in this interval, $q(t)$ will be flat-function which satisfies the estimate (2.23). 
Remark 2.3. From Theorem 2.4, we ut tain that if we have the following equation

$$
t \frac{d u}{d t}=B(t, u)
$$

and $B(t, u)$ satisfies the following condition

$$
\operatorname{Re}(B(t, u), u) \geq-C|| u(t)\left\|^{2} \ln \right\| u(t) \|^{2},
$$

then each solution of (2.24) such that

must be fiat.

$$
q(t)=\|u(t)\|^{\hat{\imath}}<1 \text { for } 0<t<\delta
$$

This is not a theorem about existence of flat solutions, but if there exists solution (2.24) such that $\|u(t)\|<1$, then $\|u(t)\|$ must be flat.

$\S 3$. On the uniqueness

In $\S \S 1-2$ we obtained results about the quasiuriqueness for solutions of equation (1.1) at the point $t=0$. It is possible to obtain some results about uniqueness for solutions of this equation.

The first and the simplest result about uniqueness was obtained in Theorem 2.1. In the special case of operator $B(t, u)$ with corlition $A$ we obtain the following statement.

Theorem 3.1. Let condition $A$ be satisfied and let for each $u(t) \varepsilon D_{A}, u(t) \neq 0$ and for each $t \in I$ the following condition be satisfied:

$$
\begin{array}{r}
\left(\left[\frac{d}{d t} A(t, u)\right] u(t), u(t)\right)>-C\{|(A(t, u) u(t), u(t))|+|| u(t) \|\} \\
\text { for every } t \in I \text { and for some constant } c \geq 0 .
\end{array}
$$

Then for each non-trivial solution $u(t)$ under those conditions, the following estimate holds

$$
\|u(t)\|>\|u(t)\|\left(\frac{t}{t_{0}}\right)^{\mu} \text { for } t<t
$$

where constant $\mu \geq 0$ depencis on $C$ from (3.1) and $u(t)$ itself.

Proof. Proof of this theorem is similar to proof of Theorem 1.3 and is based on the following statement (like Lemma 1.1).

Lemma 3.1. Let $1(t)$ be a twice differentiable non-trivial function in the interval $I$, satisfying the following second-order differential inequality

$$
D^{2} 1(t)+t a(t)[D 1(t) \mid+t b(t)>0, t \quad I
$$

where $D=t \frac{d}{d t}, a(t), b(t)$ are non-negative functions, bcunded in $I$. Then

$$
1(t)>1\left(t_{0}\right)+2 v \ln \frac{t}{t_{0}}+2 \mu l n \frac{t}{t_{0}}
$$

where constant $v \geq 0$ depending on $a(t), b(t)$ only and constant $\mu \geq 0$ depending on $a(t), b(t)$ and $u(t)$ itself. From this lemma, we obtain the estimate (3.2). From estimate (3.2) we obtain that the following function

$$
t^{-\mu}\|u(t)\|
$$

is a strongly monotonic in the interval $I=(0,1]$ and this function is decreasing ir this interval. From this we have the classical uniqueness under these conditions for every $t=t_{0}>0$.

Theorem 3.2. Under conditions of Theorem 3.1, we have the classical uniqueness for solutions of equation (1.1) in the following sense:

i) if $u\left(t_{0}\right) \neq 0$ for $t_{0} \varepsilon I=(0,1]$, then $u(t) \neq 0$ in the interval $I=(0,1]$. 
ii) if $u\left(t_{0}\right)=0$ for ${ }_{C} \in I=(0,1]$, then $u(t)=c$ ir. the inter ici $1=(0,1]$. Froof. ii) follows from $i$, arra i) follows immediateiy from the fact that furiction (3.5) is strongly monotonic. If $A(t, u)$ satisfies the foliowing cordition

i) $(A(t, u) v, v)$ is strongly positive for each $u^{\prime}(t)$ : $\square_{A}$, and for each $v \varepsilon H$ $|i v| \mid=1$ and for each $t \varepsilon I$, or $(A(t, u) v, v) \geq \varepsilon>0, \varepsilon$, micj' be dependent on $u(t)$.

ii) $\left(A(t, u j v, v)\right.$ has bounded first derivatives for each $u(t) \in D_{A}$ and for each $v$ with $\|v\|=1$ arid for each $t \& J$ with respect to $t$, maximuri of those derivatives depends on $\mathrm{U}(\mathrm{t})$, then the classical uniqueness takes place in the interva? I in the serise of Theorem 3.2 or the following statement is true.

Theorem 3.3. Let $B(t, u)$ sàtisfy condition $A$ and $A(t, u)$ satisfy the conditions i) - $i i)$ of the previous discussion. Then, the coriclusion of Theorem 3.2 is true.

Proof. Let $u(t)$ be a non-trivial solution of equation (1.1) urider conditions of this theoren. Then there exists constant $C \geq 0$ which depends on $u(t)$ maybe, such that the following is true for each $v$ with $\| v ! !=1$

$$
\left(\frac{d}{d t}[A(t, u)] v, v\right)+C(A(t, u) v, v)>0 \text { in the interval I. }
$$

.This cundition is encugh for obtaining secono-order differential iriequality (3.3) and by using Lemma 3.1 , we have that the function (3.5) is strongly decreasing in the interval I. From this, statenient of our theorem follows immediately.

Remark 3.1. Recall that in this section, we require for the classical uniqueness of solutions of equation (1.1) in the intervai I, more tough conditiors than for the quasiuniqueness at the point $t=C$ in $\S \S 1-2$. REcall also, that we do not have here complete results about the classical uniqueness in the interval $I$, as Theorem 2.3 about the quasiuniqueness at the point $t=0$.

In the case of condition (2.6) it is possible to obtain also the classical uniqueness. Namely, let condition (2.6) be satisfied and let $u(t)$ be a non-trivial solution of equation (1.1). Then, if

we have for $q(t)$ the following equation

$$
q(t)=\|u(t)\|^{2} \text {, }
$$

$$
t \dot{q}(t)=2 q\left(A\left(t, q^{\frac{1}{2}} v\right) v, v\right) \text { where } u(t)=q^{\frac{1}{2}}(t) v(t) .
$$

From (2.6) we obtain that the following form

$$
\left(A\left(t, q^{\frac{1}{2}} v\right) v, v\right) \rightarrow 0 \text { as } t \rightarrow 0
$$

and for each $\varepsilon>0$ there exists $\varepsilon>0$ such that

$$
\left|\left(A\left(t, q^{\frac{1}{2}} v\right) v, v\right)\right| \leq \varepsilon \text { whenever } t<\delta
$$

with $\varepsilon$, $\delta$ depending on $u(t)$, then from this we have for $q(t)$ the following inequality

$$
\frac{t \dot{q}}{q} \leq \varepsilon
$$

or

$$
\frac{\dot{q}}{q} \leq \frac{\varepsilon}{\mathrm{t}}
$$

or

$$
\ln \frac{q(t)}{q\left(t_{0}\right)} \leq \varepsilon \ln \frac{t}{t_{0}}
$$


or

$$
q(t) \leq q_{i}\left(t_{0}\right) \exp ^{\varepsilon} \ln \frac{t}{t_{C}}=q\left(t_{0}\right)\left(\frac{t}{t_{C}}\right)^{\varepsilon}
$$

ald since this is true for each $\varepsilon>0$, from this discussion we obtain that the following statement is true.

Theorem 3.4. Let $B(t, u)$ satisfy the condition $A$ and $A(t, u)$ satisfy the condition (2.6). If for each $u(t) \in D_{A}$ the following function

$$
(A(t . u) v, v) \rightarrow 0 \text { as } t \rightarrow 0 \text { for each } v \text { with }\|v\|=1 \text {, }
$$

then the conclusion of Theoren 3.2 is true.

Prout. The proof follows immediate?y from our estimate (3.8).

६4. The non-degentrate case

Let us consider the followirg non-degenerate non-linear equation in the Hilbert space H:

$$
\frac{d u}{d t}=B(t, u(t)) \text { where } t \in \bar{I}=[1,+\infty) \text {. }
$$

$u(t$; for each $t \varepsilon \bar{I}$ is an element of $H$ and has derivative with respect to t. $B(t, u(t))$ is nor-linear map from $H$ to $H$ with aomain $D_{B} \cdot D_{B}$ is the dense subset of $H$, and for each $t \varepsilon \bar{I}$ and for each $u(t) \varepsilon D_{B}, B(t, u(t))$ is an element of $H$ also. $B(t, u(t))$ is rot necessarily bounded. $H$ is a Hilbert space with. scalar produce (.,.) and with norm $\|$.!| correspondingly.

As in the degenerate situation, operator $B(t, u)$ is called a smocth operator of self-adjoint type if the scaiar product

$$
(B(t, u), u)
$$

is real and has derivative with respect to $t$ for each $t \varepsilon \bar{I}$ and for each $u(t) \varepsilon D_{B}$. After the change of variable $t$ by formula

$$
s=e^{-t}
$$

we obtain from equation (4.1) the following equation

$$
\frac{d u}{d s}=B(s, u) \text { for } s \in I=(0,1] \text {. }
$$

The equation (4.2) is the equation of the type (1.1) and because of this, it is possible to rewrite our results for equation (4.1). It is easy to see that in this situation, the following class of functions plays the role of the flat functions:

Class A:

$$
\left\{f(t), t \in \bar{I}: \text { for each } c>0 \quad e^{C t} f(t) \rightarrow 0 \text { as } t \rightarrow+\infty\right\}
$$

In this situation, we have the following type of quasiuniqueness.

Definition 4.1. We say that the quasiuniqueness takes place for solutions of equation (4.1) at the point $t=+_{\infty}$, if the following statement is true:

If $u(t)$ is a solution of equation (4.1) and $\|u(t)\|$ belongs to Class $A$ or, in other words: for each $c>0$, e $\|u(t)\| \rightarrow 0$ as $t \rightarrow+\infty$, then $u(t) \equiv 0$ for $t>N$ for some $N<\infty$. Now it is possible to rewrite all theorems of $\S \S 1-3$ for the case of equation (4.1).

Theorem 4.1. Let $u(t)$ be a solution of equation (4.1) such that

$$
\begin{aligned}
& \left(\frac{d}{d t}[B(t, u(t)], u(t))+\|B(t, u(t))\|^{2}-\frac{2 B(t, u(t)), u(t))^{2}}{\|u(t)\|^{2}} \geq\right. \\
& -C e^{-t}\left[|(B(t, u(t)), u(t))|+\|u(t)\|^{2}\right] \text { for some constant } C \geq 0 .
\end{aligned}
$$


Then the following is true:

$$
\text { i) }\|u(t)\| \geq M\left\|u\left(t_{0}\right)\right\| t^{-\mu t}
$$

where constant $\mu \geq 0$ depends on $C$ from (4.3) aria $u(t)$ itscif.

ii) If $u(\tau)$ belongs to class $A$, then $u(t) \equiv 0$ ir the interval $\bar{I}=[1,+\infty)$, or the quasiuniqueness takes place for solution of equation (4.1) unaer those conditions at the point $t=+\infty$.

Proof. The proof follows from Theorem 1.i.

Remark 4.2. In the linear case, these type of theorems and these type of estimates were obtained first by Agmon-Nirenberg [1,2]. It is fossible also to study the special case of equation (4.1) under condition. A. From the Theorem 1.3, we have that the following statement is true.

Theorem 4.2. Let $u(t)$ be a solution of equation (4.1) under condition $A$ such that

$$
\left(\left[\frac{d}{d t} A(t, u)\right] u(t), u(t)\right) \geq-C \cdot e^{-t}\left[|(A(t, u) u, u)|+\|u(t)\|^{2}\right]
$$

for some constant $C \geq 0$. Then the conclusion of Theorem 4.1 is true.

It is possible also to study equation (4.1) under condition (1.26) and if $A(t, u)$ as a function of $t$ satisties the frillowing condition

$$
\left(\frac{d}{d t}[A(t, u)\lrcorner v, v \geq-C e^{-t}(A(t, u) v, v)\right.
$$

for each $v$ with $\|v\|=1$ with some constant $C \geq 0$, then the conclusion of Theorem 4.1 is true also.

From (4.6) we have, if $f(t)$ denotes $(A(t, u) v, v)$ as function of $t$, that

$$
f^{\prime}(t)=-C e^{-t} f(t)
$$

or

$$
\frac{f^{\prime}(t)}{f(t)} \geq-C e^{-t}
$$

or

$$
\ln \frac{f(t)}{f\left(t_{0}\right)} \geq-c t_{0}^{\int e^{-\tau} d \tau}=C e^{-\tau} t_{0}^{t}=c\left(e^{-t}-e^{-t} 0\right)
$$

or

$$
f(t) \geq f\left(t_{0}\right) e^{C e^{-t}} e^{-C e^{-t}}=f\left(t_{0}\right) e^{C\left(e^{-t}-e^{-t} 0\right)}
$$

or

$$
f(t) \geq M f\left(t_{0}\right) e^{C e^{-t}}
$$

From (4.7) we obtain that the following function

$$
f(t) e^{-C e^{-t}}
$$

is a monotonic and it is not decreasing in the interval $\bar{I}=[1,+\infty)$. For example, if $f(t)$ is not decreasing in the interval $\bar{I}=[1,+\infty)$, this condition is enough to satisfy the condition (4.7) or the condition (4.8) with $C=0$.

From this discussion and Theorem 1.4, we obtain that the following statement is true.

Theorem 4.3. Let conditions $A$ and $(1.26)$ be satisfied and let $(A(t, u) v, v)$ be a monotonic function with unique minimum for $t=+_{\infty}$ for each $u(t)$ from class $A$ and for each $v$ with $\|v\|=1$. Let $u(t)$ be a solution of equation (4.1) from class $A$. Then $u(t) \equiv 0$ in the interval $\bar{I}$. In other words, the quasiuniqueness takes place 
under those conditions for solutions of equation (4.1) at the point $t=+\alpha$. From the previous discussion ana Theorem 1.5, we obtain that the following theorem is true.

Theorem 4.4. Let conditions $A$ and (1.26) be satisfied. Let $(A(t, u) v, v)$ as function of $t$ for each $u(t) \varepsilon\left[{ }_{A}, u(t)\right.$ from class $A$ satisties the following ccridition:

$(A(t, u) v, v ;$ is monotonic with local minimum at the point $t=+\infty$ in some neighborhood of the $t=+\infty$ with respect to $t$ in the interval $\bar{I}=[1,+\infty)$ for each $u(t)$ from class $A$ and for each $v$ with $\|v\|=1$. If $u(t)$ is a solution oi equation (4.1) from class $A$, then $u(t) \equiv 0$ in the interval $(N,+\infty)$ with $N$ depending on $u(t)$ itself. From Theorem 2.1, we obtain that the following statement is truc.

Thecrem 4.5. Let $u(t)$ be a solution of equation (4.1) under condition (2.1). If $\|u(t)\|+0$ as $t \rightarrow+\infty$, then $u(t)=0$ in the interval $\bar{I}=[1,+\infty)$. In this situation, we have classical uniqueness at the point $t=+\infty$. Let now $A(t, u)$ satisfy the following condition

$$
(A(t, u) v, v) \rightarrow 0 \text { as } t \rightarrow+\infty
$$

for, each $u(t)$ from class $A$. and for each $v \varepsilon H$ with $\|v\|=1$. From Theorem 2.2, we obtain that the following statement is true.

Theorem 4.6. Let $A(t, u)$ satisfy the condition (4.9). Then if $u(t)$

is a solution of equation (4.1) from class $A, u(t)$ is a trivial in the interval $(N,+\infty)$ with $N<\infty$ depending on $u(t)$ itself. From the previous theorems and Theorem 2.3, we obtain that the following statement is true.

Theorem 4.7. Let $B(u)$, not dependent on $t$, satisfy condition $A$ for $u$ from class $A$ or $(A(t, u) v, v)=f(t)$ a function from $c^{l} H_{1}(\bar{I})$ with respect to $t$, $t>N$ for each $u(t)$ from class $A$ and for each $v(t)$ with $\|v\|=1$. Let $u(t)$ be a solution of equation (4.1) from class $A$. Then $u(t) \equiv 0$ in the interval $(N,+\infty)$ with $N<+\infty$ depending on $u(t)$ itself.

Proof.

i) If $(A(t, u) v, v) \rightarrow 0$ as $t \rightarrow 0$ this statement follows from Theorem 4.6;

ii) If $(A(t, u) v, v) \leq 0$ in the interval $(N,+\infty)$, this statement follows from Theorem 4.5 .

iii) If $\left.(A(t, u) v, v)\right|_{u=0}>0$ in the interval $(N,+\infty)$ and $\left.\left(\frac{d}{d t}[A(t, u)] v, v\right)\right|_{u=0}$ $\geq 0$ in this interval, this statement follows from Theorem 4.4 since condition (4.7) is satisfied with $C=0$.

iv) If $\left.(f(u) v, v)\right|_{u=0}>0$ in the interval $(N,+\infty)$ and $\left.\left(\frac{d}{d t}[A(u)] v, v\right)\right|_{u=0}<0$ in this interval, this statement follows from condition (4.7) since in this situation in the derivative $\frac{d}{d t}[A(u)]$ we will have terms with $u_{t}^{1}(t)$ and from having $u(t)$ belong to class $A$, it follows that in the interval $(N,+\infty)$ we can choose constant $C$ such that (4.7) will be satisfied in some neighborhood of the point $t=+\infty$. In this situation, $C$ depends on $u(t)$ itself. Recall that interval $(N,+\infty)$ depends also on $u(t)$.

Remark 4.2. It is possible to rewrite our theorems from $\S 3$ about uniqueness for the case of equation (4.1) and to prove these using Lemma 3.1 and monotonicity of functions of type

$$
e^{\mu t}|| u(t) \| \mid
$$

in the interval $I=[1,+\infty)$ with some $\mu>0$. 
\$5. Examples

Let us consider the followirg equation

$$
t \frac{\partial u}{\partial t}=F\left(t, u, \frac{\partial u}{\partial \lambda}, \frac{\partial^{2} u}{\partial x^{2}}, \ldots, \frac{\partial^{n}}{\partial x^{n}} \frac{u}{m}\right) u
$$

where $t \varepsilon I=(0,1]$ and $x \in \Omega \subset R^{1}, \Omega$ is compact or compact 1 manifold, $F$ is a $C^{1}$-function with respect to all variables $\left(t, u, z_{j}, \ldots, z_{m}\right)$ for all values of these variables. $F$ is real-values function.

Fror. Theorem 2.3 we obtain that for equation (5.1) the quasiuniqueness takes place at the point $t:=0$ for classical solutions. If function $F$ belongs to $C^{1}$ iri some neighborhood of the origin only, we obtain from Theorem 2.3 that the quasiuniqueness takes place at the point $t=0$ too.

From flatness of solution $u(t, x)$ of equation (5.1) we cbtain that there exists a neighborhood $[0, \varepsilon]$ with $\varepsilon>0$ depending on $u(t)$ itself and in this neighborhood $u(t)=C$ for classical solutions.

Reniark 5.1. Recall that this statement is true for classical solutions cf equation (5.1) only, because in this situation we have that

$$
\frac{\partial^{i} u}{\partial x^{i}} \rightarrow 0 i=1, \ldots, m \text { as } t \rightarrow 0
$$

for flat furction $u(t, x)$. In this case also from flatness of $u(t, x)$ follows the flatness of $\partial^{i} u / \partial x^{i}$ for $i=1, \ldots . m$. And if $\Omega$ is compact, it is possible to choose for each $\delta>0$ the neighborhood of the point $t=0$ the interval $[0, \varepsilon]$ such that

$$
\left|\frac{\partial^{i} u}{\partial x^{i}}\right|<\delta \text { for } i=0,1, \ldots, m \text { if } t<\varepsilon .
$$

2. Let us consider the following equation

$$
t \frac{\partial u}{\partial t}=F\left(t, x, u, \frac{\partial u}{\partial x}, \ldots, \frac{\partial^{m} u}{\partial x^{m}}\right)
$$

where $t \in I=(0,1]$ and $x \in \Omega \quad R^{1}$ and compact or $\Omega$ is a compact manifold, $F$ is a $c^{1}$-function $w i$ th respect to all variables $\left(t, x, u, z_{1}, \ldots, z_{m}\right)$ for all values of these variables or for some neighborhood of the point $(0, x, 0, \ldots 0)$ for each $x \in s_{6}$. If $F$ does not change sign for each $x \in s$, or in other words, if one of the following conditions is satisfied:

i) $\min _{x \in \Omega}|F(0, x, 0, \ldots, 0)|>0$,

ii) for each $x \varepsilon \Omega F\left(t, x, u, z_{1}, \ldots, z_{m}\right) \leq 0$ for all values $t, u, z_{1}, \ldots, z_{m}$ such that $t \leq \varepsilon,|u| \leq \varepsilon, \ldots,\left|z_{m}\right| \leq \varepsilon$,

iii) $\quad F\left(t, x, u, z_{1}, \ldots, z_{m}^{m}\right) \rightarrow 0$ wherever $|u| \cdot\left|z_{1}\right| \cdot\left|z_{2}\right| \cdot \ldots \cdot\left|z_{m}\right| \rightarrow 0$ and $|u|^{2}+\left|z_{1}\right|^{2}+\ldots+\left|z_{m}\right|^{2} \leq \delta>0$, then the quasiuniqueness takes place at the point $t=0$ for classical solutions of this equation. From flatness of solution $u(t)$ of eauation (5.2), we obtain that there exists $\varepsilon>0$ depending on $u(t)$ itself such that $u(t)=0$ in the interval $[0, \varepsilon]$.

3. Let us consider the following equation

$$
t \frac{\partial u}{\partial t}=F\left(t, u, \frac{\partial u}{\partial t_{i}}, \frac{\partial^{2} u}{\partial x_{i_{1}}{ }^{\partial x}{ }_{i_{2}}}, \ldots, \frac{\partial^{m} u}{\partial x_{i_{1}} \cdots \partial x_{i_{m}}}\right) u(t, x)
$$

where $t \varepsilon I=(0,1], x \in s_{i} \subset R^{n}$ and compact or $s_{i}$ is compact manifold, $i_{k}=1, \ldots n$ 
ului $f$ is a $r^{i}$-function wit , spect to ill variabies $\left(t, u, z, \ldots, z_{n} ;\right.$ for dil values rif these variables. $F$ is real-values function.

From Theoren i..3, vie obtain that for equation (5.3) the quasiuniqueness takt: place at the point $t=0$ for $c$ assicicl solutiors. If function $F$ belongs to $c^{1}$ in sone neighborhcond of the crigin only, we obtain frori Theoren 2.3 that the cuasiuniqueness takes $\mu$ ?ace at the point $t=0$ tou.

Frorl fidtness of solution $u(t, x)$ of equation (5.3), we get that there exists $\varepsilon, 0$ dependent on $u(t, x)$ itself such that $u(t)=C$ in the intervat $[0, \varepsilon]$.

Remark 5.2. It is possible to ot tain resuits about the classical unigueness of equition (5.1)-(5.4) in the interval I, tut for this we must require that function $F$ catisfies one of the following conditions:

i) $F \leq 0$ for all values of all variables.

ii) $F \geq \delta$, C for all values of all variables.

Ther irom results of $\S 3$ we obtain that the classical uriqueness takes place in the selise of Theorem 3.2, or

i) $i 1 \quad u\left(t_{0}\right) \neq 0$ for $t_{0} \varepsilon i=(0,1]$, then $u(t) \neq 0$ in the interval

ii) if $u\left(t_{0}\right)=0$ for $t_{0} \& I=(0,1]$, then $\left.u ! t\right)=0$ in the intervai $I=(0,1 j$ including $t=0$.

Recall that $i)-i i)$ are like the standard conditions for uniqueness in the linear cose.

4. Let us consider the following equation

$$
\left.t \frac{\partial u}{\partial t}=F\left(t, x_{i}\right\} \ldots, x_{n}, u, \frac{\partial u}{\partial t_{i}}, \frac{\partial^{2} u}{\partial x_{i} \partial x_{j}}, \ldots, \frac{\partial^{m} u}{\partial x_{i_{1}} \ldots \partial x_{i_{m}}}\right) u(t, x)
$$

where $t \in I=(0,1], x \in s_{i} \subset R^{n}$ and compact $i_{k}=1, \ldots n$ and $F$ is a real $c^{1}$-furiction with respect to $\dot{c} ! 1$ variables $\left(t, x, u, z_{1}, \ldots z_{n}\right)$ for all values of these variabies for some neighborhcod of the point $(0, x, 0, \ldots 0)$ for each $x \in \Omega$. If function $F$ satisfies one of the conditions i)-iii) from example 2 , then the quasiuniqueness takes place at the point $t=0$ for classical solutions of equation (5.4). From flatness of classical solution $u(t)$ of equation (5.4), we obtain that there exists $\varepsilon>0$ dependent on $u(t)$ itself such that $u(t)=0$ in the interval $[0, \varepsilon]$.

5. Let us consider the following equation

$$
\frac{\partial u}{\partial t}=F\left(t, u, \frac{\partial u}{\partial x_{i}}, \frac{\partial^{2} u}{\partial x_{i_{1}} \partial x_{i_{2}}}, \ldots, \frac{\partial^{m} u}{\partial x_{i_{1}} \ldots \partial x_{i_{m}}}\right) u(t, x)
$$

where $t \in \bar{I}=[1,+\infty), x \in s_{i} R^{n}$ and $s i$ is conpact or compact manifold $i_{k}=1, \ldots, n$ and $F$ is a $c^{1}$-function with respect to all variables $\left(t, u, z_{1}, \ldots, z_{n}\right)$ for all values of these variables. $F$ is a real-values function.

From Theorem 4.7 we cbtain that for equation (5.5), the quasiuniqueness takes place at the point $t=+\infty$ for classical solutions. If function $F$ belongs to $C^{1}$ in some neighborhood of the point $(t, 0, C, \ldots, 0)$ for each $t>N_{0}<+\infty$, we obtain from Theorem 4.7 that the quasiuniqueness takes place at the point $t=+\infty$ also.

If solution $u(t)$ of equation (5.5) belongs to class $A$, we get that there exists $\varepsilon>0$ dependent on $u(t)$ itself such that $u(t)=0$ in the interval $(1 / \varepsilon,+\infty)$. 
6 . Let us consider the foliowing equation

$$
\frac{\partial u}{\partial t}=F\left(t, x_{1}, \ldots, x_{n}, u, \frac{\partial u}{\partial x_{i}}, \ldots, \frac{\partial^{m} u}{\partial x_{i_{1}} \ldots \partial x_{i_{m i}}}\right) u(t, x)
$$

where $t \in \bar{i}=[1,+\infty), x \in \Omega \subset R^{n}$ and compact of $s_{0}$ is a compact manifold, $i_{k}=1, \ldots n$ and $F$ is a $C^{1}$-function with respect to all variables for all

volues of variables or for sumie neighborhood of the point $(t, \lambda, 0, \ldots .0)$ for each $x \varepsilon 0$, ard for each $t>\frac{1}{\varepsilon}$ for $\varepsilon>0$.

If $F$ satisfies one of the conditions of exanple 2, then the quasiuniqueness takes place at the point $t=+\infty$ for classical solutions of equation (5.6). If sclution $u(t, x)$ of equation (5.6) belongs to class $A$, we get that there exists $\varepsilon>0$ dependent $c r . u(t)$ itself such that $u(t)=0$ in the interval $(1 / \varepsilon,+\infty)$.

Remark 5.3. It is possible to obtain also results about the classical uniqueness of equation (5.5)-(5.6) in the interval $\bar{I}$, as we obtained in Remark 5.2. References

i. S. Agmon and L. Nirenberg, "Properties of solutions of ordinary differential equations in Banach space, Conm. Pure Appl. Math., 16(1963), pp. 121-239.

2. S. Agmon, Unicite et convexite dans les problemes differentieles, Montreal, 1966.

3. V. Schuchman, Dissertation, The Weizmann Institute of Science, Rehovot, 1981.

4. V. Schuchman, The behavior of solutions of non-linear differential equations in Hilbert space II, North Texas State University, Denton, 1981.

5. V. Schuchman, The behavior of solutions of non-linear differential equations in Hilbert space I, North Texas State University, Denton, 1981. 


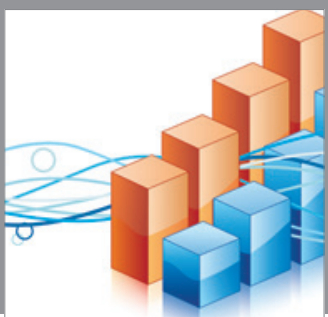

Advances in

Operations Research

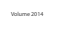

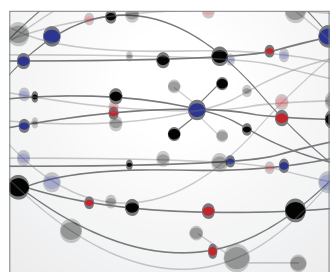

\section{The Scientific} World Journal
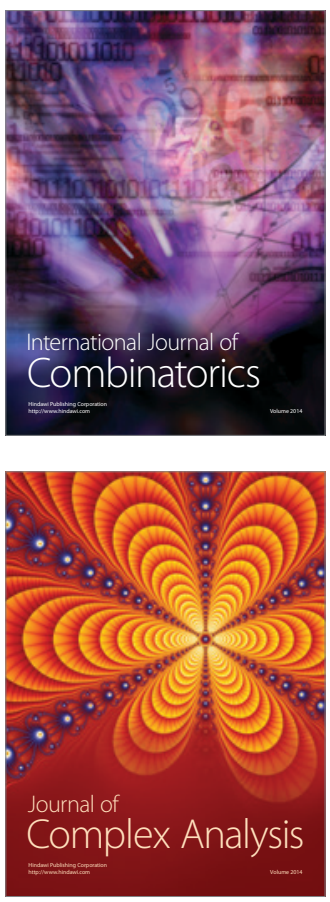

International Journal of

Mathematics and

Mathematical

Sciences
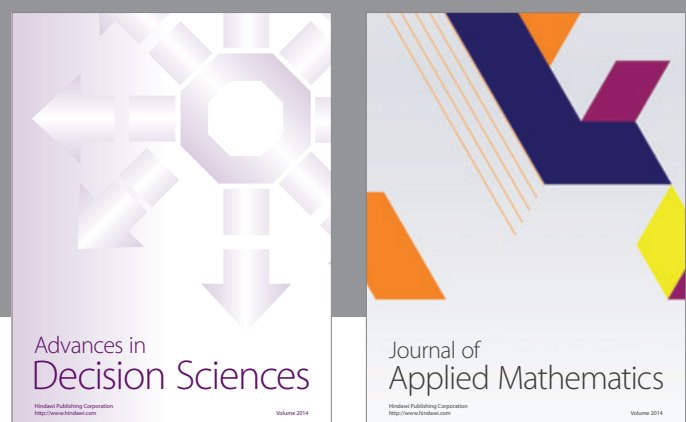

Journal of

Applied Mathematics
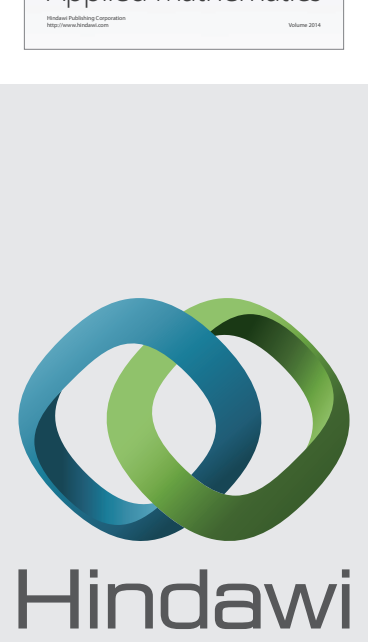

Submit your manuscripts at http://www.hindawi.com
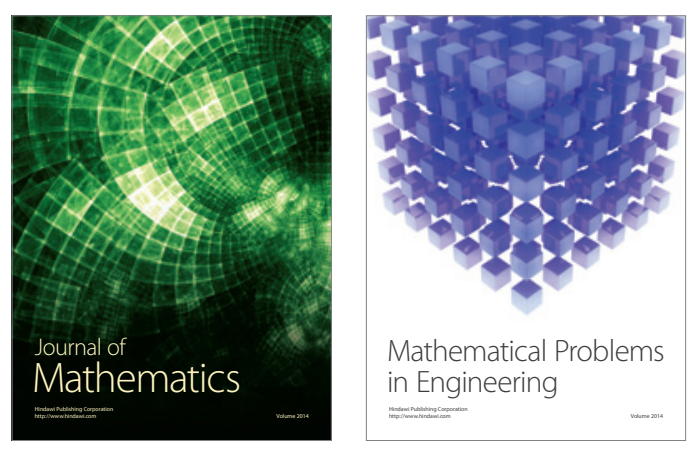

Mathematical Problems in Engineering
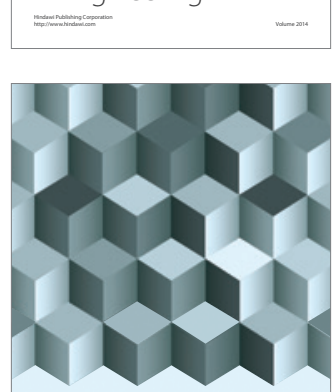

Journal of

Function Spaces
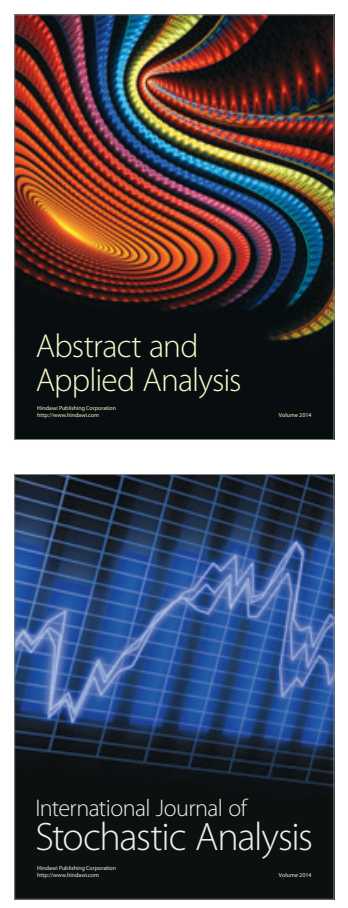

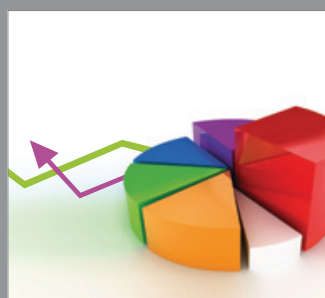

ournal of

Probability and Statistics

Promensencen
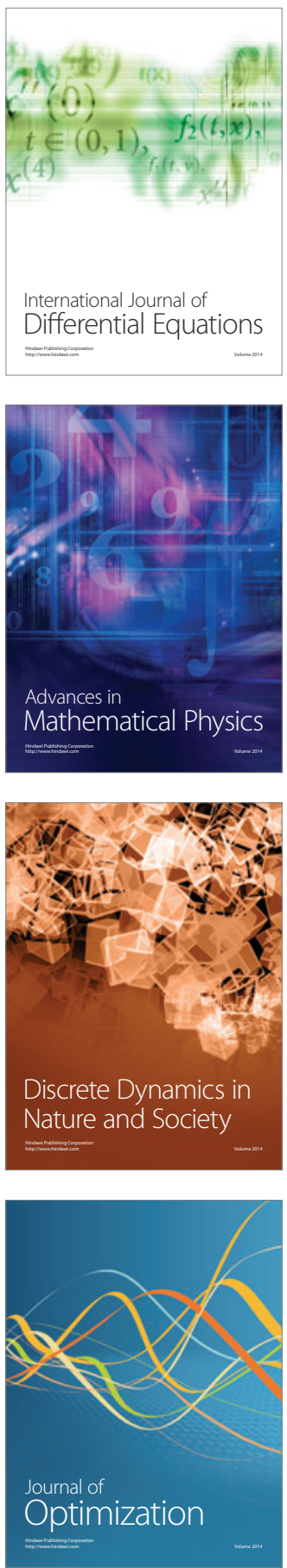